\title{
Poster: Supporting JavaScript Experimentation with BUGSJS
}

\author{
Béla Vancsics*, Péter Gyimesi*, Andrea Stocco ${ }^{\dagger}$, Davood Mazinanian ${ }^{\dagger}$, Árpád Beszédes*, Rudolf Ferenc*, Ali Mesbah ${ }^{\dagger}$ \\ *University of Szeged, Hungary ${ }^{\dagger}$ University of British Columbia, Canada \\ \{vancsics, pgyimesi, beszedes, ferenc\}@inf.u-szeged.hu \\ \{astocco,dmazinanian, amesbah\}@ece.ubc.ca
}

\begin{abstract}
In our recent work, we proposed BUGSJS, a benchmark of several hundred bugs from popular JavaScript serverside programs. In this abstract paper, we report the results of our initial evaluation in adopting BUGSJS to support an experiment in fault localization. First, we describe how BUGSJS facilitated accessing the information required to perform the experiment, namely, test case code, their outcomes, their associated code coverage and related bug information. Second, we illustrate how BUGSJS can be improved to further enable easier application to fault localization research, for instance, by filtering out failing test cases that do not directly contribute to a bug.

We hope that our preliminary results will foster researchers in using BUGSJS to enable highly-reproducible empirical studies and comparisons of JavaScript analysis and testing tools.
\end{abstract}

Keywords-JavaScript, bug database, real bugs, fault localization, benchmark, reproducibility, BUGSJS.

\section{INTRODUCTION AND MOTIVATION}

JavaScript is a popular language for developing highly interactive web applications, recently also adopted for serverside code [1]. However, JavaScript is an error-prone language, due to its asynchronous, dynamic, and loosely typed nature. Research has focused in recent years on devising automated testing techniques for JavaScript, among which automatic fault localization [2], or root cause analysis of bugs [3].

Novel software analysis and testing techniques are typically evaluated through empirical methods (e.g., controlled experiments), which rely on various software-related artifacts, such as source code, test suites, and descriptive bug reports. Comparing the efficacy of these techniques on a common centralized benchmark is imperative to ensure reliability and replicability. Unfortunately, in the JavaScript domain, our survey of the previous studies showed an extensive heterogeneity in the used evaluation subject systems [4].

To fill this gap, we have proposed BUGSJS, a benchmark of several hundreds manually-validated bugs from 10 popular JavaScript programs, along with comprehensive bug reports and one or more test cases that demonstrate the bugs [4]. These artifacts can be used for devising and evaluating analysis and testing techniques for JavaScript, facilitated through an API that allows accessing and executing the faulty and fixed versions of the programs and the corresponding tests. While several benchmarks of bugs have been proposed [5, 6, 7, 8, 9. 10], to our knowledge, BUGSJS is the first benchmark of detailed, descriptive, and curated programs and bug reports for JavaScript.

In this extended abstract, we report the results of using the BUGSJS benchmark and infrastructure to perform an experiment in fault localization, along with some lessons learned and future directions.

\section{BugsJS}

BUGSJS includes 453 reproducible bugs from 10 popular Node.js server-side JavaScript programs that adopt the Mocha testing framework. We have mined these projects from GitHub with the following criteria: (i) popularity: stargazers count $\geq 100$, (ii) maturity: number of commits $>200$, and (iii) recency: year of the latest commit $\geq 2017$. Table I shows the characteristics of the selected projects. The last column shows the initial number of bug candidates we considered for inclusion in the benchmark, from which several had to be removed due to reasons explained below.

TABLE I: Subjects included in BUGSJS

\begin{tabular}{lrrrrr}
\hline & kLOC & Stars & Commits & Forks & $\begin{array}{c}\text { Candidate } \\
\text { Bugs }\end{array}$ \\
\hline Bower & 16 & 15,290 & 2,706 & 1,995 & 10 \\
ESLint & 240 & 12,434 & 6,615 & 2,141 & 559 \\
Express & 11 & 40,407 & 5,500 & 7,055 & 39 \\
Hessian.js & 6 & 104 & 217 & 23 & 17 \\
Hexo & 17 & 23,748 & 2,545 & 3,277 & 24 \\
Karma & 12 & 10,210 & 2,485 & 1,531 & 37 \\
Mongoose & 65 & 17,036 & 9,770 & 2,457 & 56 \\
Node-redis & 11 & 10,349 & 1,242 & 1,245 & 25 \\
Pencilblue & 46 & 1,596 & 3,675 & 276 & 18 \\
Shield & 20 & 6,319 & 2,036 & 1,432 & 10 \\
\hline Total & 444 & 137,493 & 36,791 & 21,432 & 795 \\
\hline
\end{tabular}

The bugs included in BUGSJS are automatically extracted from the issue tracking systems of the included programs. To ensure artefacts traceability, we included closed issues which were assigned with a specific bug label in the issue tracker.

For each bug, BUGSJS contains the full textual description of the bug reports and their metadata, along with the developers' discussions. Moreover, we have included the corresponding bug-fixing commit in order to keep the source code changes required to fix each bug. All bug-fixing commits in 
TABLE II: Manual and dynamic validation statistics

\begin{tabular}{|c|c|c|c|c|c|c|c|c|c|c|c|}
\hline & 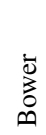 & $\begin{array}{l}\vec{\Xi} \\
\overrightarrow{\vec{n}} \\
\text { II }\end{array}$ & 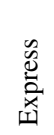 & 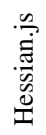 & & 节 & 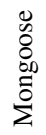 & 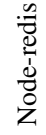 & $\begin{array}{l}\frac{y}{0} \\
\overline{0} \\
0 \\
0 \\
0\end{array}$ & $\frac{\overrightarrow{\frac{\pi}{d}}}{\frac{\pi}{\pi}}$ & 픙 \\
\hline Candidate bugs & 10 & 559 & 39 & 17 & 24 & 37 & 56 & 25 & 18 & 10 & 795 \\
\hline After manual validation & 8 & 382 & 33 & 13 & 13 & 26 & 41 & 11 & 8 & 7 & 542 \\
\hline After dynamic validation & 3 & 333 & 27 & 9 & 12 & 22 & 29 & 7 & 7 & 4 & 453 \\
\hline
\end{tabular}

BUGSJS have been validated and cleaned, both manuallyby multiple authors-and dynamically, to ensure that the bugs and their fixes were relevant, isolated, and reproducible.

Relevance means that the bug-fixing commits are actually fixing the bug described in the issue, rather than, for example, implementing a new feature. Isolation means that the bugfixing code exclusively aim at fixing the bug, and no other changes (e.g., refactorings) are interleaved within it. Isolation is particularly important in domains where bug-fixing changes should be clearly identifiable, hence must be cleaned (e.g., training machine learning models to learn the fixes from source code to devise automated program repair techniques). Bugs in BUGSJS are also reproducible, since we have manually extracted the test cases that demonstrate each bug. Each set of such tests was executed on the buggy revision (i.e., dynamic validation) to ensure their relevance to the bug, and can be leveraged, for instance, to devise novel automated test generation techniques. The results of our validation of the bugs in BUGSJS are reported in Table II.

We have used the GitHub's fork feature to include the entire history of the projects in BUGSJS. Each bug in BUGSJS is tagged to five source code revisions, as follows:

1) The parent commit of the revision in which the bug was fixed (i.e., the buggy revision);

2) A revision with the original bug-fixing changes (including the production code and the newly added tests);

3) A revision with only the tests introduced in the bug-fixing commit, applied to the buggy revision;

4) A revision only containing the production code changes introduced to fix the bug, applied to the buggy revision;

5) A revision containing both the cleaned fix and the newly added tests, applied to the buggy revision.

BugSJS OOrganization
\begin{tabular}{|c|c|c|l|l|}
\hline \multicolumn{1}{|c|}{$\begin{array}{l}\text { Subject\#1 } \\
\text { Fork }\end{array}$} & \multicolumn{1}{|c|}{$\begin{array}{c}\text { Subject\#N } \\
\text { Fork }\end{array}$} & $\begin{array}{c}\text { bug dataset } \\
\text { Repository }\end{array}$ & $\begin{array}{c}\text { docker environment } \\
\text { Repository }\end{array}$ \\
\cline { 1 - 3 } $\begin{array}{l}\text { Source code } \\
\text { Tests } \\
\text { Cleaned patches } \\
\text { Tagged bug fixes }\end{array}$ & $\ldots$ & $\begin{array}{l}\text { Source code } \\
\text { Tests } \\
\text { Cleaned patches } \\
\text { Tagged bug fixes }\end{array}$ & $\begin{array}{l}\text { Utility framework } \\
\text { Bug statistics } \\
\text { Test commands } \\
\text { Bug report data }\end{array}$ & Pre-built environment \\
\hline \multicolumn{1}{|c|}{ Forked } & Forked \\
\hline $\begin{array}{c}\text { Subject\#1 } \\
\text { Original repository }\end{array}$ & $\begin{array}{c}\text { Subject\#N } \\
\text { Original repository }\end{array}$ & \\
\hline
\end{tabular}

Fig. 1: BUGSJS architecture
BUGSJS consists of two other components (see Figure 1): a Docker image that provides a runtime environment, and a bug dataset. The dataset can be queried through an API that supports the following commands: (i) info: printing out information about a given bug, (ii) checkout: checking-out the source code, (iii) test: running all tests and retrieving the overall test suite coverage (iv) per-test: running each test individually and retrieving the per-test coverage. These commands facilitate the use of BUGSJS for other researchers to a large extent.

More details about the characteristics of BUGSJS, its architecture, and our design choices can be found in our full paper [4]. The interested reader can find more information on BUGSJS and access the benchmark on our website:

$$
\text { https://bugsjs.github.io/ }
$$

\section{Possible Use CASes}

BUGSJS can be useful to support experimentation in several testing research areas, some of which we list next.

Regression testing. More than $25 \mathrm{k}$ test cases included in BUGSJS can facilitate various regression testing studies, e.g., test prioritization, software oracles, or automated test repair.

Bug prediction. The source code and various information pertaining to a large set of bugs available in BUGSJS can be used to construct bug prediction models. The availability of both cleaned and uncleaned bug-fixing patches in the dataset can allow assessing the sensitivity of the proposed models to noise.

Automated program repair. The manually-cleaned patches available in BUGSJS can be used as learning examples for patch generation in novel automated program repair for JavaScript. Also, BUGSJS provides an out-of-the-box solution for automatic dynamic patch validation.

Bug localization. BUGSJS contains pointers to the natural language bug descriptions/discussions for several hundreds of bugs. Devising NLP-based techniques for formulating natural language queries that describe the observed bugs available in BUGSJS is just one sample use case. Also, other approaches to fault localization such as Spectrum-Based Fault Localization can benefit from readily available sets of test cases, detailed code coverage information, test case outcomes and bug positions. We chose this use case as a preliminary example of use of our dataset, which we explain next.

\section{Sample Use Case: Fault localization}

We used BUGSJS to support an ongoing experimentation on fault localization (FL) [2], a well-established research area that can largely benefit from a dataset of catalogued bugs. To evaluate FL algorithms, we need validated bugs, their fixes, as well as tests to demonstrate the existence of bugs. In our use case, we considered Spectrum-Based Fault Localization (SBFL), a popular FL technique which captures run-time information, such as detailed code coverage, in order to monitor the dynamic behaviour of the software. Then, by associating the test case outcomes with appropriate dynamic information, 
we can identify the potentially faulty code fragments in the program.

One widely-used program spectra in SBFL is the one which is based on the covered/uncovered statements and methods during test execution. To successfully apply this technique, we require: (i) test coverage information, (ii) tests results, and (iii) modified source code (e.g., statements or methods that were modified during the fixing).

BUGSJS's API provides easy access to all such information. High coverage and non-extreme test-method ratio are also important for the efficiency of FL algorithms. The dataset contains an average of 9327 tests, 1562 methods, 8941 LOC, and features $96 \%$ method-level coverage and $\approx 37: 6$ testmethod ratio.

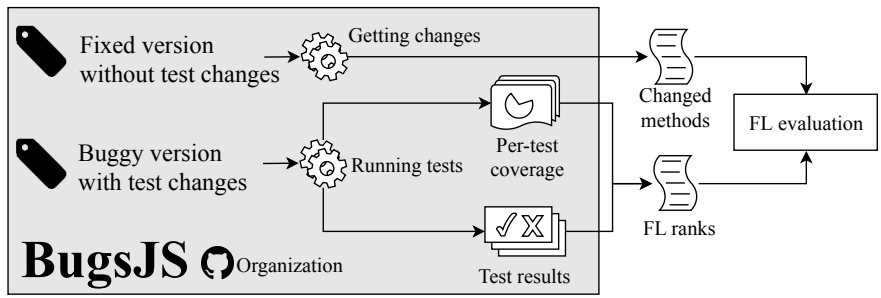

Fig. 2: Fault localization process

The overall FL process using BUGSJS is shown in Figure 2. First, the per-test command retrieves which methods are covered by a test, which provides an overview of the full, method-level coverage of the subject program. Second, the test command executes all tests and saves the results. The difference between the buggy and the fixed version determines which files were changed by a fix-commit, that is, the location of the bug in the source code.

A common approach in FL is to provide a ranking score to order program entities (e.g., methods) based on their likeliness to be buggy. The ranking score is typically a function of four values for a given method $m$, all of which can be easily obtained from BUGSJS:

a) $m_{e p}$ : number of passing tests covered by $m$

b) $m_{e f}$ : number of failing tests covered by $m$

c) $m_{n p}:$ number of passing tests not covered by $m$

d) $m_{n f}$ : number of failing tests not covered by $m$

In this work, we used Tarantula [11], a popular ranking score defined as follows:

$$
\operatorname{Tarantula}(m)=\frac{\frac{m_{e f}}{m_{e f}+m_{n f}}}{\frac{m_{e f}}{m_{e f}+m_{n f}}+\frac{m_{e p}}{m_{e p}+m_{n p}}}
$$

\section{A. Preliminary results: bug-fix patterns and ranks}

We applied SBFL with Tarantula technique to the subject program Hessian.js, which includes nine (9) bugs.

As part of the analysis and classification of BugsJS bugs [4], we categorized them based on recurring bug-fix patterns proposed by Pan et al. [12]. By comparing the results of this categorization and the ranking scores of the fixing methods provided by Tarantula, we expect to gather insights on how different types of bugs can be successfully localized.
TABLE III: Hessian. js bug-fix patterns and Tarantula ranks

\begin{tabular}{ccll}
\hline Bug \# & Rank & Method & Pattern(s) [12] \\
\hline 5 & 1 & lib/v2/encoder.js:(anonymous_3) & \\
2 & 2 & lib/v2/decoder.js:(anonymous_15) & IF-RMV \\
9 & 2 & lib/v1/decoder.js:(anonymous_20) & SQ-RMO \\
3 & 3 & lib/v1/encoder.js:(anonymous_21) & IF-APCJ \\
8 & 3 & lib/utils.js:(anonymous_3) & IF-CC \\
6 & 4,5 & ib/v2/decoder.js:(anonymous_11) & IF-APC \\
4 & 7 & lib/v1/encoder.js:(anonymous_18) & IF-CC \\
7 & 7 & lib/v1/encoder.js:(anonymous_19) & CF-CHG \\
1 & 7,5 & lib/v2/encoder.js:(anonymous_11) & MC-DNP, SQ-AMO \\
1 & 7,5 & lib/v2/encoder.js:(anonymous_12) & MC-DNP, SQ-RMO \\
6 & 8 & lib/v1/decoder.js:(anonymous_20) & IF-APC \\
2 & 9 & lib/v1/decoder.js:(anonymous_20) & IF-CC \\
1 & 41,5 & lib/v2/encoder.js:Encoder & CF-ADD \\
5 & 56,5 & lib/v2/encoder.js:(anonymous_4) & IF-APC \\
\hline
\end{tabular}

For all nine bugs from the Hessian.js project, we determined, using the Tarantula score, the ranks for each method affected by the bug-fixes, then we assigned bug-fix patterns to these methods. Table III lists the bug ID in BUGSJS, the rank obtained from the Tarantula score, the corresponding methods in the bug-fix and the associated bug-fix patterns.

The first method (lib/v2/encoder.js: (anonymous_3)) does not have any patterns assigned because the fix only contains an assert statement which is not among the patterns proposed by Pan et al. [12]. We can observe that two methods falling within the IF-CC pattern (lib/utils.js:(anonymous_3), lib/v1/encoder.js:(anonymous_18)) have generally better ranks. In these cases, the fix involved adding or modifying existing IF conditions. The only exception is lib/v1/decoder.js: (anonymous_20), where the fix only involved removing a condition. The ranks of fixes falling within the IF-APC $(J)$ pattern are rather different, whereas the occurrence of other patters is not significant. Thus, further experiments with more bug information (for instance, those of the other projects available in BUGSJS) are required to draw conclusions about the correlation between bug patterns and fault localization techniques.

\section{B. Preliminary results: ranking of methods}

The previous section presented all bugs from the Hessian.js project. Additionally, let us consider Bug-2 of this projec 17 which contains 175 tests, 95 methods, 1040 LOC, the method-level coverage is around $98 \%$ and the test-method ratio is $\approx 35: 19$. These statistics (i.e., non-trivial number of tests and code elements with balanced ratio) enable a more reliable application of our fault localization technique.

The bug-fixing commit changed nine (9) lines of JavaScript code. Based on the location of these changes, we can precisely identify the modified methods (as BUGSJS's coverage data contain information about the starting LOC of each method). In this case, the bug-fixing commit involves

\footnotetext{
${ }^{1}$ https://github.com/BugsJS/hessian.js/releases/tag/Bug-2-fix
} 
TABLE IV: Hessian. js Bug-2 changes and scores

\begin{tabular}{lcccccc}
\hline Method & $m_{e f}$ & $m_{e p}$ & $m_{n f}$ & $m_{n p}$ & Tarantula & Rank \\
\hline anonymous_15 & 1 & 6 & 1 & 167 & $\mathbf{0 . 9 3 5 1 4}$ & $\mathbf{2}$ \\
anonymous_20 & 1 & 23 & 1 & 150 & 0.78995 & 9 \\
\hline
\end{tabular}

two methods: lib/v1/decoder.js:(anonymous_20) and lib/v2/decoder.js: (anonymous_15). Table IV reports the four metrics required to compute Tarantula values and the final scores for each of these methods. Results are ranked according to increasing Tarantula scores, hence showing which method is more likely to be buggy. In our example, anonymous_20 is ranked ninth and anonymous_15 is ranked second in the order of all methods. Since the bug-fixing commit involves multiple methods, the lowest rank associated with all changed methods determines which is the rank of the bug. In this case, it will be two, that is the rank of anonymous_15. Since the identified buggy element also included fixes to an if-construct, this is aligned with our observation from the previous section.

\section{An additional observation}

Unfortunately, we observed that programs can also include failing tests that are not related to the investigated bug. The unrelated tests can interfere with the evaluation of FL algorithms, as such they they must be filtered out. One possible way to do it is by comparing the test results of the buggy and fixed versions: tests that fail in both cases are irrelevant to the examined bug. For example, Hessian. js contains six (6) such tests, on average.

\section{CONCLUSions And Future Work}

In this abstract paper we have sketched our on-going work on the BUGSJS benchmark which has the ultimate purpose of representing a consolidated resource available to researchers in the areas of software analysis and testing for JavaScript. We have also reported an example of possible experiment in fault localization for JavaScript using BUGSJS.

We hope our framework can foster further research in this domain, and that can be used to conduct highly-reproducible empirical studies in various areas such as regression testing, bug prediction, and fault localization.

As part of our ongoing and future work, we plan to include more subjects (and corresponding bugs) to the benchmark. Also, we plan to implement easier filtering of such irrelevant test cases, so as to reduce the false positive ration when performing experiments in fault localization. Our long-term goal is to also include client-side JavaScript web applications in BUGSJS. Furthermore, we are planning to develop an abstraction layer to allow easier extensibility of our infrastructure to other JavaScript testing frameworks.

\section{REFERENCES}

[1] S. Alimadadi, A. Mesbah, and K. Pattabiraman, "Understanding asynchronous interactions in full-stack JavaScript," in Proc. of 38th International Conference on Software Engineering (ICSE), 2016.

[2] W. E. Wong, R. Gao, Y. Li, R. Abreu, and F. Wotawa, "A survey on software fault localization," IEEE Transactions on Software Engineering, vol. 42, no. 8, 2016.

[3] F. S. Ocariza, K. Bajaj, K. Pattabiraman, and A. Mesbah, "A Study of Causes and Consequences of ClientSide JavaScript Bugs," IEEE Transactions on Software Engineering, vol. 43, no. 2, pp. 128-144, Feb 2017.

[4] P. Gyimesi, B. Vancsics, A. Stocco, D. Mazinanian, Árpád Beszédes, R. Ferenc, and A. Mesbah, "BugJS: A benchmark of javascript bugs," in Proceedings of 12th IEEE International Conference on Software Testing, Verification and Validation (ICST), 2019. [Online]. Available: https://bugsjs.github.io/paper/ICST19.pdf

[5] H. Do, S. Elbaum, and G. Rothermel, "Supporting controlled experimentation with testing techniques: An infrastructure and its potential impact," Empirical Softw. Engg., vol. 10, no. 4, pp. 405-435, Oct. 2005.

[6] R. Just, D. Jalali, and M. D. Ernst, "Defects4J: A database of existing faults to enable controlled testing studies for Java programs," in Proc. of 2014 International Symposium on Software Testing and Analysis, 2014.

[7] C. Le Goues, N. Holtschulte, E. K. Smith, Y. Brun, P. Devanbu, S. Forrest, and W. Weimer, "The ManyBugs and IntroClass benchmarks for automated repair of $\mathrm{C}$ programs," IEEE Transactions on Software Engineering (TSE), vol. 41, no. 12, pp. 1236-1256, December 2015.

[8] N. Dmeiri, D. A. Tomassi, Y. Wang, A. Bhowmick, Y.-C. Liu, P. Devanbu, B. Vasilescu, and C. RubioGonzalez, "BugSwarm: Mining and Continuously Growing a Dataset of Reproducible Failures and Fixes," in Proc. of 41st International Conference on Software Engineering (ICSE), 2019.

[9] G. Fraser and A. Arcuri, "Sound empirical evidence in software testing," in Proc. of 34th International Conference on Software Engineering (ICSE), 2012.

[10] A. Gkortzis, D. Mitropoulos, and D. Spinellis, "VulinOSS: A dataset of security vulnerabilities in opensource systems," in Proc. of 15th International Conference on Mining Software Repositories, 2018.

[11] J. A. Jones and M. J. Harrold, "Empirical evaluation of the tarantula automatic fault-localization technique," in Proceedings of the 20th IEEE/ACM International Conference on Automated Software Engineering, ser. ASE '05, 2005, pp. 273-282.

[12] K. Pan, S. Kim, and E. J. Whitehead, "Toward an understanding of bug fix patterns," Empirical Software Engineering, vol. 14, no. 3, pp. 286-315, Jun 2009. 